\title{
ASPECTOS DISCRIMINANTES ENTRE USUÁRIOS E NÃO USUÁRIOS DA INTERNET BANDA LARGA
}

\author{
Maria Aparecida Gouvêa \\ Doutora em Administração pela Universidade de São Paulo - USP \\ Professora da Universidade de São Paulo - USP \\ magouvea@usp.br
}

\section{Leonardo Felipe Japur de Sá}

Bacharel em Engenharia de Produção pela Universidade de São Paulo - USP

leofjsa@gmail.com

\section{Daielly Melina Nassif Mantovani}

Doutora em Administração pela Universidade de São Paulo - USP

daimantovani@terra.com.br

\section{RESUMO}

A internet tem se destacado como meio de comunicação nas mais diversas formas. Isso não seria possível se a tecnologia de transmissão de dados via internet não tivesse se desenvolvido a ponto de permitir velocidades adequadas para essas aplicações. No mercado de pequenas empresas, contudo, constata-se uma parcela significativa de usuários de internet discada que ainda não aderiram à banda larga. Este estudo teve dois objetivos: testar a dimensionalidade e a fidedignidade de um modelo de aceitação tecnológica no contexto de adoção de banda larga e identificar os fatores do modelo que apresentam diferenças significativas entre os usuários e não usuários desse serviço. Foram identificadas diferenças significativas para os fatores intenção de comportamento, influência social e condições facilitadoras. Dentre esses fatores, os que apresentam média negativa (condições facilitadoras e intenção de comportamento) para os não usuários de banda larga podem representar barreiras de adoção de internet banda larga por parte do potencial cliente.

Palavras-chave: Aceitação tecnológica; Banda larga; Pequenas empresas. 


\section{INTRODUÇÃO}

O mercado de internet caracteriza-se, entre outros fatores, por ainda apresentar um crescimento acelerado, de forma que não se sabe exatamente como se comportará como um mercado maduro. Segundo a Telebrasil (n.d.), o mercado de acesso banda larga no Brasil apresentou crescimento de $14 \%$ em 2009 em relação a 2008 (Gráfico 1).

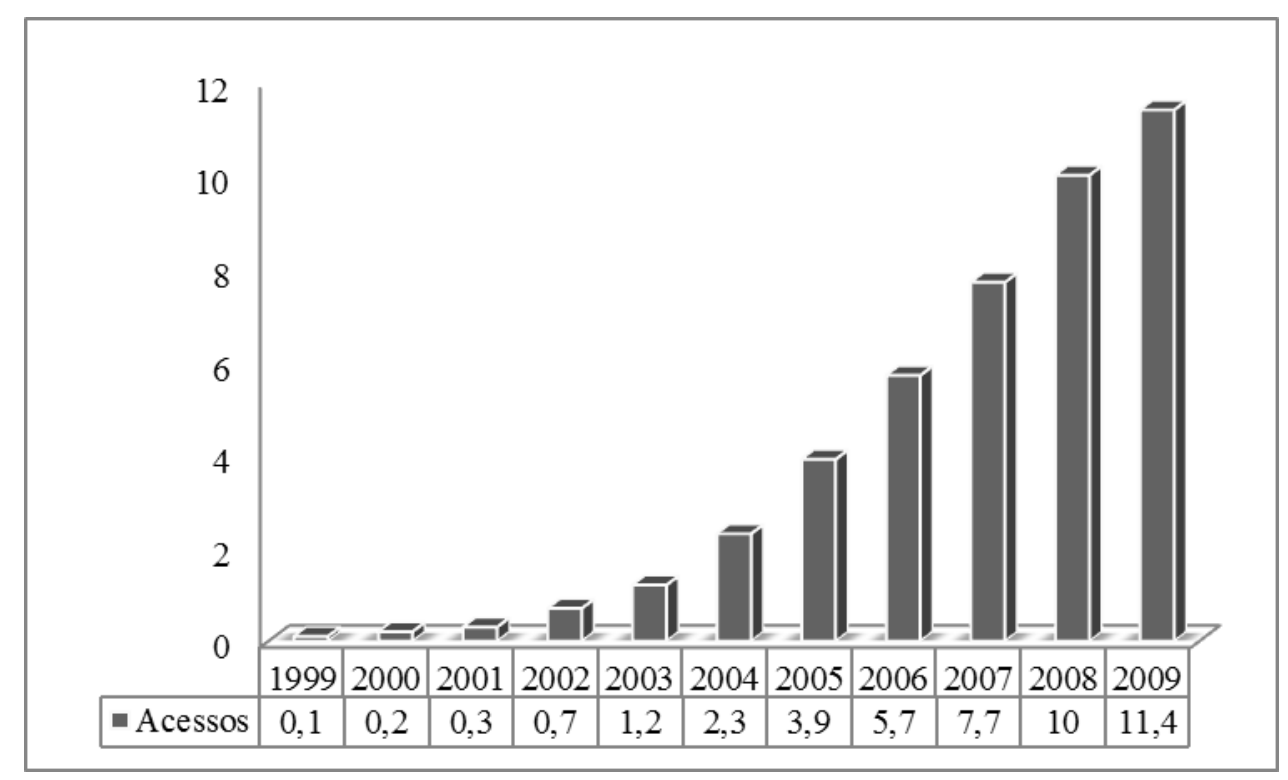

Gráfico 1 - Evolução anual do número de acessos banda larga no Brasil (em milhões) Fonte: Telebrasil (n.d.).

$\mathrm{Na}$ totalidade do mercado no Brasil, os serviços de internet banda larga estavam presentes em 12 milhões de domicílios em 2009. O plano nacional de banda larga, um projeto fomentado pelo governo, pretende ter, até 2014, 40 milhões de domicílios com esse tipo de acesso. Vale ressaltar que, apesar do forte avanço do acesso à internet no Brasil, os problemas de qualidade ainda são iminentes, já que apenas 3,1\% da população possui acesso de alta capacidade (mais de $8 \mathrm{mb} / \mathrm{s}$ ); 41,8\% dos acessos advêm de conexões de baixa velocidade (até $512 \mathrm{~kb} / \mathrm{s}$ ); 43,8\% dos acessos possuem conexão com velocidade entre $512 \mathrm{~kb} / \mathrm{s}$ e $2 \mathrm{mb} / \mathrm{s}$ e $10,3 \%$ possuem conexão com velocidade de $2 \mathrm{mb} / \mathrm{s}$ a $8 \mathrm{mb} / \mathrm{s}$ (Comitê Gestor de Internet no Brasil, n.d.).

Parte desse forte avanço da banda larga no Brasil pode ser atribuída à necessidade de conexões mais velozes que é provocada pela popularização de aplicações que envolvem alto volume de tráfego, geralmente de som ou imagem, tais como download de arquivos de música, voz sobre IP (VoIP), fotos digitais, vídeos etc.

Revista de Administração e Inovação, São Paulo, v. 9, n.3, p. 195-212, jul/set. 2012. 
Dos volumes de acessos em 2010 no Brasil, a parcela atribuída a empresas fica em torno de 20\%, evidenciando o predomínio dos acessos residenciais. Por esse motivo, há interesse dos fornecedores de banda larga em desenvolver produtos e alocar esforço comercial focados para as corporações.

Considerando-se as pequenas empresas, depara-se com uma carência de produtos de banda larga específicos. As suas necessidades normalmente são definidas em uma situação intermediária entre dois públicos: residencial e grandes corporações.

Em um extremo, têm-se produtos para o público residencial por meio, principalmente, de tecnologia DSL ou cabo (oferecidos, respectivamente, por operadoras de telefonia fixa e de televisão por assinatura). Para grandes empresas, no outro extremo, há outros produtos (denominados link IP) que garantem maior estabilidade de conexão (o que implica maior confiabilidade no serviço), porém com custos em torno de cinco vezes mais altos para a mesma velocidade de acesso. Para as pequenas empresas, as concessionárias de telefonia fixa, operadoras de TV a cabo ou outros fornecedores criaram evoluções dos planos residenciais. No entanto, esses planos apresentam pouca diferenciação de benefícios em relação ao original, apesar de haver diferenças significativas no custo final para o cliente.

Esse posicionamento de produtos pode gerar uma barreira potencial para adoção de banda larga em pequenas empresas.

O crescimento acelerado do mercado de banda larga no Brasil observado nos últimos anos, bem como a falta de produtos de banda larga específicos para pequenas empresas, motivaram a realização deste estudo cujos objetivos definem-se como:

- testar a dimensionalidade e a fidedignidade de um modelo de aceitação tecnológica no contexto de adoção de banda larga;

- identificar os fatores do modelo que apresentam diferenças significativas entre os usuários e não usuários de banda larga.

\section{FUNDAMENTAÇÃO TEÓRICA}

A fundamentação teórica deste trabalho tem foco em aceitação de tecnologia e segue a linha de pesquisa de F. Davis e V. Venkatesh, culminando na Teoria Unificada de Aceitação e Uso de Tecnologia (Venkatesh, Morris, Davis, \& Davis, 2003). 
Sistemas de computador não podem melhorar o desempenho organizacional se não são usados. Infelizmente, resistência a sistemas para 'o usuário final' por executivos e profissionais é um problema generalizado. Para melhor prever, explicar e incrementar a aceitação do usuário precisamos entender melhor porque as pessoas aceitam ou rejeitam computadores (Davis, Bagozzi, \& Warshaw, 1989, p. 982, tradução nossa).

Foi com essas palavras que Davis et al. (1989) iniciaram seu texto para justificar o desenvolvimento do Modelo de Aceitação de Tecnologia (TAM - Technology Acceptance Model). Para melhor compreendê-las, no entanto, é importante relembrar que, em 1989, o uso de PC's e sistemas de informação pelo usuário final (e não apenas por técnicos de informática) ainda era algo muito recente. Hoje em dia, obviamente o contexto é bastante diferente. Mas os fundamentos propostos no TAM ainda continuam válidos, inclusive para aplicações em um conceito mais amplo de tecnologia (como ilustração, vale observar que em Davis et al., 1989, a validação do modelo foi baseada na aceitação de um software de edição de texto).

Na década seguinte (anos 1990), outros modelos e teorias foram também publicados buscando identificar os fatores que levam à adoção ou não de tecnologia até que Venkatesh et al. (2003) formularam a Teoria Unificada de Aceitação e Uso de Tecnologia (que passará a ser referida como UTAUT-Unified Theory of Acceptation and Use of Technology) a partir da consolidação de conceitos de oito desses modelos e teorias. Neste estudo, a UTAUT será adaptada para avaliar a aceitação e o uso de internet banda larga.

Venkatesh et al. (2003) propuseram uma consolidação de oito modelos e teorias para gerar a UTAUT, baseados em construtos que agregam a essência dos modelos e teorias que a compõem (Figura).

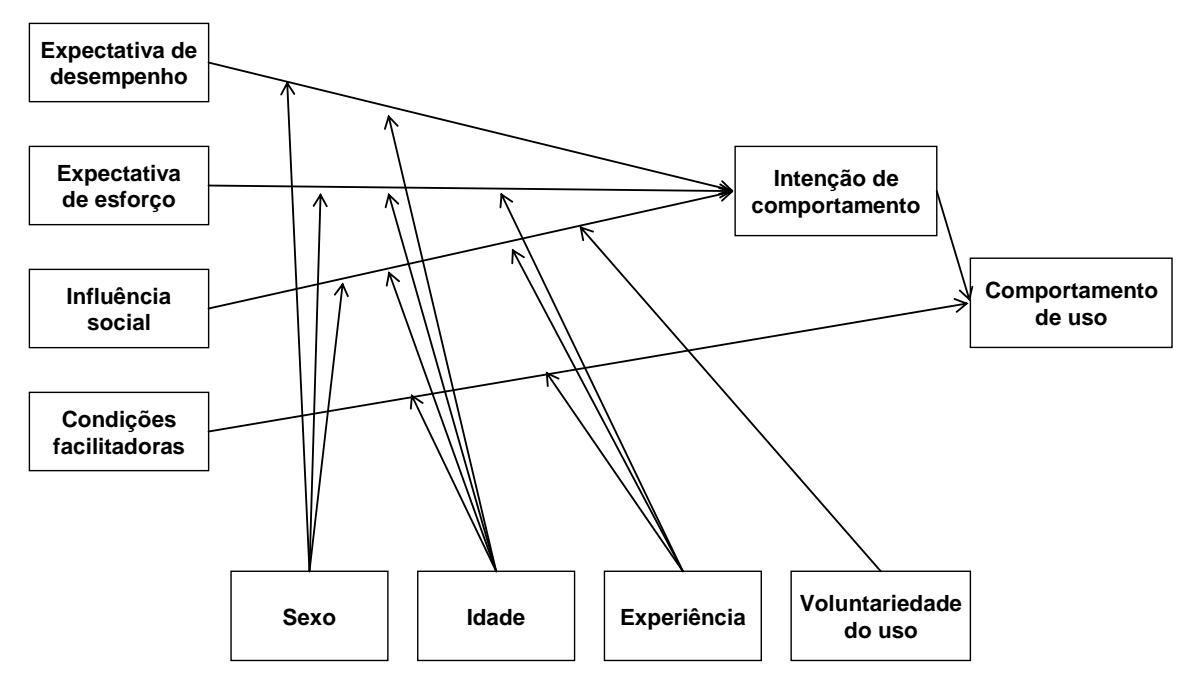

Figura 1 - A "Teoria Unificada de Aceitação e Uso de Tecnologia" Fonte: Venkatesh et al. (2003, p. 447). 
Nessa teoria, os autores apresentam quatro construtos como determinantes diretos da intenção de comportamento e do comportamento de uso: (i) expectativa de desempenho, (ii) expectativa de esforço, (iii) influência social e (iv) condições facilitadoras. A definição e origem de cada um desses determinantes constam no Quadro 1.

O construto de intenção de comportamento, presente na teoria como mediador do comportamento de uso, tem sua origem na Teoria da Ação Racionalizada. Esse construto define o grau em que o indivíduo sente-se motivado a adotar o comportamento em questão (com base na expectativa de desempenho, expectativa de esforço e na influência social), independentemente das condições que viabilizam esse comportamento (que, segundo essa teoria, são limitadas pelas condições facilitadoras).

\begin{tabular}{|c|c|c|}
\hline Construto & \begin{tabular}{|l|} 
Definição \\
\end{tabular} & Origem \\
\hline $\begin{array}{l}\text { 1. Expectativa de } \\
\text { desempenho }\end{array}$ & $\begin{array}{l}\text { Grau em que um indivíduo acredita que o uso do } \\
\text { sistema vai ajudá-lo a atingir ganhos no } \\
\text { resultado do trabalho }\end{array}$ & $\begin{array}{l}\text { - Utilidade Percebida (TAM/TAM2 e } \\
\text { DTPB) } \\
\text { - Motivação extrínseca (MM) } \\
\text { - } \quad \text { Adequação da função (MPCU) } \\
\text { - Vantagem relativa (IDT) } \\
\text { - Expectativa de resultados (SCT) }\end{array}$ \\
\hline $\begin{array}{l}\text { 2. Expectativa de } \\
\text { esforço }\end{array}$ & Grau de facilidade associada ao uso do sistema & $\begin{array}{l}\text { - } \text { Facilidade de uso percebida } \\
\text { (TAM/TAM2) } \\
\text { - } \quad \text { Complexidade (MPCU) } \\
\text { - } \quad \text { Facilidade de uso (IDT) } \\
\end{array}$ \\
\hline $\begin{array}{l}\text { 3. Influência } \\
\text { social }\end{array}$ & $\begin{array}{l}\text { Grau em que um indivíduo percebe que outras } \\
\text { pessoas importantes acreditam que ele deveria } \\
\text { usar o novo sistema }\end{array}$ & $\begin{array}{l}\text { - Norma subjetiva (TRA, TAM2, TPB, } \\
\text { DTPB) } \\
\text { - Fatores sociais (MPCU) } \\
\text { - Imagem (IDT) } \\
\end{array}$ \\
\hline $\begin{array}{l}\text { 4. Condições } \\
\text { facilitadoras }\end{array}$ & $\begin{array}{l}\text { Grau em que um indivíduo acredita que existe } \\
\text { uma infraestrutura organizacional e técnica para } \\
\text { suportar o uso do sistema }\end{array}$ & $\begin{array}{l}\text { - Controle percebido do comportamento } \\
\text { (DTPB) } \\
\text { - Condições facilitadoras (MPCU) } \\
\text { - Compatibilidade (IDT) } \\
\end{array}$ \\
\hline
\end{tabular}

Siglas:TRA: Theory of Reasoned Action (Ajzen \& Fishbein, 1980); TPB: Theory of Planned Behavior (Ajzen, 1991); TAM: Technology Acceptance Model (Davis et al., 1989); IDT: Innovation Diffusion Theory (Rogers, 1995); DTPB: Decomposed Theory of Planned Behavior (Taylor \& Todd, 1995); MM: Motivational Model (Davis, Bagozzi, \& Warshaw, 1992); MPCU: Model of PC Utilization (Thompson, Higgins, \& Howell, 1991); SCT: Social Cognitive Theory (Bandura, 1986, Compeau \& Higgins, 1995; Compeau, Higgins, \& Huff, 1999).

Quadro 1 - Os fatores principais da UTAUT

Fonte: Venkatesh et al. (2003, p. 447, 450-451, 453).

Por fim, os autores definem alguns mediadores dos determinantes diretos, incluindo aspectos pessoais (sexo e idade) e aspectos com relação à situação de uso (experiência e voluntariedade de uso).

Os oito modelos e teorias que compõem a UTAUT são apresentados de maneira resumida, com o objetivo de melhor contextualizar sua formação (Quadro 2). Para maior detalhamento desses fundamentos, recomenda-se consultar as obras originais de cada um dos modelos e teorias. 


\begin{tabular}{|c|c|}
\hline Modelo / Teoria & Principais construtos \\
\hline $\begin{array}{l}\text { - TRA: Teoria da } \\
\text { Ação Racionalizada }\end{array}$ & $\begin{array}{l}\text { - Atitude em relação ao comportamento: "sentimento positivo ou negativo } \\
\text { de um indivíduo sobre a execução de um determinado comportamento" } \\
\text { (Fishbein \& Ajzen, 1975, p. 216, tradução nossa). } \\
\text { - Norma subjetiva: "percepção de um indivíduo de que a maioria das } \\
\text { pessoas que são importantes para ele pensa que deveria ou não deveria adotar } \\
\text { o comportamento em questão" (Fishbein \& Ajzen, 1975, p. 302, tradução } \\
\text { nossa). }\end{array}$ \\
\hline $\begin{array}{l}\text { - TPB: Teoria do } \\
\text { comportamento } \\
\text { planejado }\end{array}$ & $\begin{array}{l}\text { - Atitude em relação ao comportamento: adaptado da TRA } \\
\text { - Norma subjetiva: adaptado da TRA } \\
\text { - Controle percebido do comportamento: “a facilidade ou dificuldade } \\
\text { percebida em executar o comportamento" (Ajzen, 1991, p. 188, tradução } \\
\text { nossa). No contexto de pesquisa de sistemas de informação, "percepções de } \\
\text { restrições internas e externas ao comportamento' (Taylor \& Todd, 1995, p. } \\
149 \text {, tradução nossa). }\end{array}$ \\
\hline $\begin{array}{l}\text { - TAM e TAM2: } \\
\text { Modelo de Aceitação } \\
\text { de Tecnologia }\end{array}$ & $\begin{array}{l}\text { - Utilidade Percebida: "grau em que uma pessoa acredita que o uso de um } \\
\text { sistema em particular aumentaria seu desempenho" (Davis, 1989, p. 320) } \\
\text { - Facilidade de uso percebida: "grau em que uma pessoa acredita que o uso } \\
\text { de um sistema em particular seria livre de esforço' (Davis, 1989, p. 320, } \\
\text { tradução nossa). } \\
\text { - Norma subjetiva: adaptado da TRA (somente no TAM2) }\end{array}$ \\
\hline $\begin{array}{l}\text { - Teoria de difusão } \\
\text { da inovação (IDT) }\end{array}$ & $\begin{array}{l}\text { - Vantagem relativa: "grau em que uma inovação é percebida como sendo } \\
\text { melhor que sua precursora" (Moore \& Benbasat, 1991, p. 195, tradução } \\
\text { nossa). } \\
\text { - Facilidade de uso: "grau em que uma inovação é percebida como de difícil } \\
\text { uso" (Moore \& Benbasat, 1991, p. 195, tradução nossa). } \\
\text { - Imagem: "grau em que o uso de uma inovação é percebido como benéfico } \\
\text { para a imagem ou status do indivíduo em seu sistema social" (Moore \& } \\
\text { Benbasat, 1991, p. 195, tradução nossa). } \\
\text { - Visibilidade: grau em que um indivíduo pode ver outros usando o sistema } \\
\text { na organização (adaptado de Moore \& Benbasat, 1991). } \\
\text { - Compatibilidade: "grau em que uma inovação é percebida como sendo } \\
\text { consistente com os valores existentes, necessidades e experiência passada dos } \\
\text { adotantes potenciais" (Moore \& Benbasat, 1991, p. 195, tradução nossa). } \\
\text { - Demonstrabilidade de resultados: "a tangibilidade dos resultados do uso } \\
\text { de uma inovação, incluindo sua observabilidade e comunicabilidade" (Moore } \\
\text { \& Benbasat, 1991, p. 203, tradução nossa). } \\
\text { - Voluntariedade do uso: "grau em que o uso de uma inovação é percebido } \\
\text { como voluntário, ou de vontade própria" (Moore \& Benbasat, 1991, p. 195, } \\
\text { tradução nossa). }\end{array}$ \\
\hline $\begin{array}{l}\text { - MM: Modelo } \\
\text { motivacional }\end{array}$ & $\begin{array}{l}\text { - Motivação extrínseca: "percepção de que os usuários vão querer executar } \\
\text { uma atividade "porque é percebida como um instrumento para alcançar } \\
\text { resultados valiosos que são distintos da atividade propriamente dita, como } \\
\text { melhoria de desempenho no trabalho, pagamento ou promoção" (Davis et al., } \\
\text { 1992, p. 1112, tradução nossa). } \\
\text { - Motivação intrínseca: "percepção de que usuários vão querer executar uma } \\
\text { atividade "sem objetivo aparente além do processo de executar a atividade por } \\
\text { si" (Davis et al., 1992, p. 1112, tradução nossa). }\end{array}$ \\
\hline
\end{tabular}




\begin{tabular}{|c|c|}
\hline Modelo / Teoria & Principais construtos \\
\hline $\begin{array}{l}\text { - C-TAM-TPB: } \\
\text { TAM combinado } \\
\text { com TPB }\end{array}$ & $\begin{array}{l}\text { - Atitude em relação ao comportamento: adaptado da TRA/TPB } \\
\text { - Norma subjetiva: adaptado da TRA/TPB } \\
\text { - Controle percebido do comportamento: } \text { adaptado da TRA/TPB } \\
\text { - Utilidade percebida: } \text { adaptado do TAM }\end{array}$ \\
\hline $\begin{array}{l}\text { - MPCU: Modelo de } \\
\text { utilização de PC }\end{array}$ & $\begin{array}{l}\text { - Adequação da função: "intensidade na qual um indivíduo acredita que } \\
\text { usar uma tecnologia pode incrementar o desempenho de seu trabalho" } \\
\text { (Thompson et al., 1991, p. 129, tradução nossa). } \\
\text { - Complexidade: baseado em Rogers e Shoemaker (1971), "grau em que } \\
\text { uma inovação é percebida como relativamente difícil de entender e usar" } \\
\text { (Thompson et al., 1991, p. 128, tradução nossa). } \\
\text { - Consequências de longo prazo: resultados que têm uma contrapartida no } \\
\text { futuro (Thompson et al., 1991, p. 129, tradução nossa). } \\
\text { - Afeição em relação ao uso: "sentimento de satisfação, prazer, depressão, } \\
\text { desgosto, desprazer, ou ódio associado por um indivíduo a um ato particular" } \\
\text { (Thompson et al., 1991, p. 127, tradução nossa). } \\
\text { - Fatores sociais: "internalização do indivíduo da cultura subjetiva de um } \\
\text { grupo de referência, e acordos específicos interpessoais que o indivíduo tem } \\
\text { com outros em situações sociais" (Thompson et al., 1991, p. 126, tradução } \\
\text { nossa). } \\
\text { - Condiçães facilitadoras: fatores objetivos no ambiente que observadores } \\
\text { concordam como facilitador de um fato. Por exemplo, devolução de itens } \\
\text { comprados on-line é facilitada quando nenhuma taxa é cobrada sobre o item } \\
\text { devolvido. Em um contexto de sistemas de informação, "provisão de suporte } \\
\text { para usuários de PC pode ser um tipo de condição facilitadora que influencia } \\
\text { na utilização do sistema" (Thompson et al., 1991, p. 129, tradução nossa). }\end{array}$ \\
\hline $\begin{array}{l}\text { - Teoria social } \\
\text { cognitiva (SCT) }\end{array}$ & $\begin{array}{l}\text { - Expectativas de resultado (desempenho): as consequências do } \\
\text { comportamento relacionadas ao desempenho. Especificamente, expectativas } \\
\text { de desempenho lidam com resultados relacionados à função; } \\
\text { - Expectativas de resultado (pessoal): as consequências pessoais do } \\
\text { comportamento. Especificamente, expectativas pessoais lidam com a } \\
\text { autoestima e o senso de cumprimento do indivíduo; } \\
\text { - Autoeficácia: julgamento do indivíduo de sua habilidade de usar tecnologia } \\
\text { (ex.: computador) para cumprir uma tarefa em particular; } \\
\text { - Afeto: gosto de um indivíduo por um comportamento em particular (ex.: } \\
\text { uso de computador); } \\
\text { - Ansiedade: ansiedade ou reações emocionais evocadas quando se trata de } \\
\text { adotar um computador (ex.: uso de um computador). }\end{array}$ \\
\hline
\end{tabular}

Quadro 2 - Modelos e teorias que originaram a UTAUT

Fonte: Venkatesh et al. (2003, p. 428 a 432).

O modelo TAM original determina que a atitude do usuário em relação à tecnologia se dá pela percepção da utilidade e da facilidade de uso de tal tecnologia. Essa atitude leva à intenção comportamental, isto é, à adoção ou não da tecnologia em questão (Wu, Cheng, Yen, \& Huang, 2011). Todavia, quando se trata do ambiente de negócios, a aceitação da tecnologia pode ocorrer até mesmo quando o indivíduo não apresenta atitude positiva, pois aquela pode impactar positivamente o desempenho e a produtividade do funcionário no trabalho. Assim, diversos autores conduziram seus estudos excluindo a dimensão atitude do modelo (Wu et al., 2011).

Revista de Administração e Inovação, São Paulo, v. 9, n.3, p. 195-212, jul/set. 2012. 
Conforme mencionado anteriormente, Venkatesh et al. (2003), ao revisar os oito modelos diferentes para aceitação tecnológica (incluindo o TAM), verificaram que a atitude tem influência significativa no comportamento quando os construtos relacionados à performance e ao esforço decorrente do uso não estiverem incluídos no modelo. Assim, os autores adaptaram o modelo incluindo e excluindo diferentes construtos, conforme as características do problema de pesquisa (Wu et al., 2011).

Desde sua criação, os modelos de aceitação tecnológica têm sido adaptados e aplicados às mais diversas áreas nas organizações. Wu et al. (2011) estudaram a adoção da internet sem fio por colaboradores no ambiente de trabalho, utilizando o modelo de aceitação tecnológica com seus construtos originais, e uma versão simplificada sem o construto atitude, em uma amostra de 198 profissionais de Taiwan atuantes em diversos setores, tais como financeiro, indústria, serviços, governo e educação. Ambos os modelos explicam a intenção de adoção da tecnologia sem fio, porém a facilidade de uso possui maior influência sobre a adoção do que a percepção de utilidade. Assim, se a tecnologia tiver uma interface de uso de difícil assimilação pelo usuário, ele buscará alternativas para realizar seu trabalho (Wu et al., 2011).

Similarmente, Teo (2011) aplicou o modelo TAM a uma amostra de 131 alunos de um curso de pós-graduação em educação de Singapura. Os resultados indicaram, pela modelagem de equações estruturais, que a intenção de adoção de tecnologia pode ser utilizada como proxy para a mensuração do uso real da tecnologia estudada (Teo, 2011).

Brown, Dennis e Venkatesh (2010) aplicaram o modelo UTAUT mesclado a construtos advindos da teoria da tecnologia da colaboração. Nesse modelo a intenção de uso é influenciada pelas características da tecnologia (presença social, imediatismo, concorrência), pelas características individuais e do grupo (experiência com tecnologia, autoeficácia), pelas características da tarefa e dos fatores situacionais (influência dos pares, influências do ambiente) (Brown et al., 2010). Primeiramente, os autores aplicaram o modelo a uma amostra de 349 usuários de mensagens de texto (SMS); em um segundo momento, o modelo foi aplicado a um grupo de usuários de um software colaborativo usado para reuniões e conferências; ambos os estudos ocorreram em instituições finlandesas (Brown et al., 2010).

Como resultado, os autores constataram no primeiro estudo que a maior presença social aumenta o imediatismo, e a maior concorrência leva à mais elevada expectativa sobre a performance e sobre o esforço necessário à adoção da tecnologia. O modelo 2, diferente do primeiro modelo, mostrou que a maior presença social só eleva a expectativa sobre a performance em tarefas de tomada de decisão; o imediatismo possui maior efeito sobre as tarefas de tomada de decisão, e a maior 
concorrência eleva a expectativa de performance apenas para tarefas que envolvam a tomada de decisão. Assim, os autores concluem que o modelo UTAUT é eficaz para a previsão da adoção tecnológica (Brown et al., 2010).

Neste estudo, a teoria UTAUT será adaptada para o contexto da adoção da tecnologia de internet banda larga visando-se à identificação de diferenças de opiniões entre usuários e não usuários dessa tecnologia.

\section{ASPECTOS METODOLÓGICOS}

Em consonância com os objetivos deste estudo, foi desenvolvida uma pesquisa quantitativa com pequenas empresas, usuárias e não usuárias de banda larga.

O público-alvo da pesquisa quantitativa é composto por pequenas empresas localizadas no estado de São Paulo. Neste estudo, definem-se como pequenas empresas as que apresentam até 49 funcionários. O critério para definição de pequeno porte refere-se ao padrão adotado pelo Serviço de Apoio às Micro e Pequenas Empresas (SEBRAE) (incluindo também microempresas), escolhido por conveniência (critérios baseados em faturamento seriam mais difíceis de levantamento na amostragem). Segundo o Instituto Brasileiro de Geografia e Estatística ([IBGE], 2003):

Não há unanimidade sobre a delimitação do segmento das micro e pequenas empresas. Observa-se, na prática, uma variedade de critérios para a sua definição tanto por parte da legislação específica, como por parte de instituições financeiras oficiais e órgãos representativos do setor, ora baseando-se no valor do faturamento, ora no número de pessoas ocupadas, ora em ambos. A utilização de conceitos heterogêneos decorre do fato de que a finalidade e os objetivos das instituições que promovem seu enquadramento são distintos (regulamentação, crédito, estudos, etc.) (IBGE, 2003, p. 17).

Para esse público, dois grupos (subamostras) foram definidos: usuários e não usuários de banda larga (no segundo caso, incluem-se usuários de internet discada e não usuários de internet).

A amostragem foi probabilística, atendendo à necessidade de generalização de resultados. Como não foi previsto nenhum critério para estratificação das amostras, foi empregada amostragem casual simples. Em cada uma das amostras (uma para cada público), foram planejadas 100 entrevistas, que correspondem a um erro amostral de $10 \%$ nas amostras individuais, ou de $7 \%$ nas duas amostras em conjunto, considerando-se um nível de confiança de 95\%. Na amostra final, chegou-se a um número ligeiramente superior de entrevistas (107 usuários de banda larga e 112 não usuários).

Revista de Administração e Inovação, São Paulo, v. 9, n.3, p. 195-212, jul/set. 2012. 
O levantamento dos dados, baseado em questões fechadas, foi realizado por telefone no período de setembro a dezembro de 2010. As empresas sorteadas que não se dispuseram a participar da pesquisa foram substituídas de maneira aleatória. Foi escolhido esse método por facilidade operacional, garantindo foco no público de interesse para este estudo.

\section{ANÁLISE DOS RESULTADOS}

Para avaliar a adequação da escala para medida dos construtos da UTAUT, foram testadas a dimensionalidade e a fidedignidade. Segundo Urdan (1995, p. 294-299):

- A dimensionalidade sintetiza as dimensões (construtos) que são representadas pelo conjunto de variáveis da escala;

- A fidedignidade de um instrumento de medida representa a capacidade deste instrumento de "[...] produzir resultados consistentes se repetidas mensurações são realizadas".

A dimensionalidade foi testada por uma análise fatorial, e a fidedignidade, pelo Alpha de Cronbach.

O questionário contém 19 variáveis para representar os cinco construtos da UTAUT. O construto intenção de comportamento, no caso dos já usuários de banda larga, foi tratado como intenção de continuar a ser usuário desse serviço.

Segundo Hair, Black, Babin, Anderson, e Tatham (2009):

Genericamente falando, a análise fatorial fornece as ferramentas para analisar a estrutura das interrelações (correlações) em um grande número de variáveis (p. ex., escores de teste, itens de teste, respostas a questionários), definindo conjuntos de variáveis que são fortemente inter-relacionadas, conhecidos como fatores (Hair et al., 2009, p. 102).

Segundo Hair et al. (2009, p. 109), a análise fatorial é pouco exigente com relação às suas premissas. Mas os autores ressaltam como precauções necessárias o tamanho da amostra e a existência de correlações entre as variáveis da análise.

Com relação ao dimensionamento da amostra: "Como regra geral, o mínimo é ter pelo menos cinco vezes mais observações do que variáveis a serem analisadas, e uma faixa mais aceitável seria uma razão de dez-para-um” (Hair et al., 2009, p. 109). No caso deste estudo, essa relação foi superada (207 casos para 19 variáveis).

Com relação às correlações entre as variáveis, três testes são recomendados: 
- Teste de esfericidade de Bartlett: "teste estatístico para a significância geral de todas as correlações em uma matriz de correlações" (Hair et al., 2009, p. 110);

- MSA (measure of sampling adequacy): "medida calculada para toda a matriz de correlação e para cada variável individual, avaliando a adequação de se aplicar a análise fatorial" (Hair et al., 2009, p. 110);

- $\quad$ KMO (Kaiser - Meyer - Olkin): representa a proporção entre as correlações entre as variáveis (que devem ser altas) e as correlações parciais (que devem ser baixas). "KMO provê uma medida de quanto os indicadores pertencem a um mesmo construto" (Sharma, 1996, p. 116, tradução nossa). Como resultado, esperam-se valores altos (Tabachnick \& Fidell, 2001, p. 589).

O método para definição da quantidade de fatores foi pela raiz latente, no qual todos os fatores resultantes têm autovalor superior a 1, conforme recomendado por Hair et al. (2009, p. 114). O método de extração foi o de componentes principais. Para facilitar a identificação das variáveis que compõem cada fator, foi utilizado o método de rotação VARIMAX.

Como medida do poder de síntese dos cinco fatores obtidos, verificou-se que foram capazes de explicar $83 \%$ da variação total das 19 variáveis da amostra.

A Tabela 1 apresenta a matriz de componentes rotacionada desta análise, indicando em qual fator cada variável foi alocada.

Tabela 1 - Matriz de componentes rotacionada

\begin{tabular}{|c|c|c|c|c|c|}
\hline Variável & 1 & 2 & 3 & 4 & 5 \\
\hline 9.1) Utilidade no trabalho & 0,304 & 0,033 & 0,804 & 0,123 & 0,263 \\
\hline 9.2) Agilidade no cumprimento de tarefas & 0,334 & 0,058 & 0,769 & 0,197 & 0,185 \\
\hline 9.3) Aumento de produtividade & 0,187 & 0,001 & 0,874 & 0,139 & 0,123 \\
\hline $\begin{array}{l}\text { 9.4) Aumento das chances de sucesso da } \\
\text { empresa/escritório }\end{array}$ & 0,190 & 0,040 & 0,778 & 0,154 & 0,109 \\
\hline 10.1) Clareza e compreensão no uso & 0,835 & 0,019 & 0,277 & 0,237 & 0,138 \\
\hline 10.2) Facilidade em tornar-se um usuário habilidoso & 0,865 & 0,025 & 0,262 & 0,247 & 0,150 \\
\hline 10.3) Facilidade de uso & 0,855 & $-0,019$ & 0,279 & 0,294 & 0,118 \\
\hline 10.4) Facilidade no aprendizado & 0,867 & $-0,046$ & 0,232 & 0,225 & 0,094 \\
\hline $\begin{array}{l}\text { 11.1) Posse de recursos necessários (verba, } \\
\text { equipamentos, etc.) }\end{array}$ & 0,104 & $-0,184$ & 0,217 & $\mathbf{0 , 7 7 5}$ & 0,155 \\
\hline $\begin{array}{l}\text { 11.2) Posse de conhecimento específico para operar } \\
\text { com internet banda larga }\end{array}$ & 0,285 & $-0,026$ & 0,091 & 0,822 & 0,194 \\
\hline $\begin{array}{l}\text { 11.3) Compatibilidade com outros sistemas da } \\
\text { empresa/escritório }\end{array}$ & 0,253 & $-0,027$ & 0,134 & 0,820 & 0,149 \\
\hline $\begin{array}{l}\text { 11.4) Disponibilidade de suporte técnico interno } \\
\text { para caso de dificuldades }\end{array}$ & 0,274 & $-0,049$ & 0,158 & 0,826 & 0,084 \\
\hline $\begin{array}{l}\text { 12.1) Opinião de pessoas que influenciam suas } \\
\text { decisões }\end{array}$ & $-0,022$ & 0,875 & 0,020 & $-0,036$ & 0,031 \\
\hline
\end{tabular}

Revista de Administração e Inovação, São Paulo, v. 9, n.3, p. 195-212, jul/set. 2012. 
Aspectos discriminantes entre usuários e não usuários da internet banda larga

\begin{tabular}{l|c|c|c|c|c}
\hline Variável & $\mathbf{1}$ & $\mathbf{2}$ & $\mathbf{3}$ & $\mathbf{4}$ & $\mathbf{5}$ \\
\hline $\begin{array}{l}\text { 12.2) Opinião de pessoas importantes para você } \\
\text { 12.3) Apoio do(s) principal(is) responsável(is) pela } \\
\text { administração da empresa/escritório }\end{array}$ & $-0,023$ & $\mathbf{0 , 9 1 1}$ & $-0,016$ & $-0,068$ & $-0,043$ \\
\hline $\begin{array}{l}\text { 12.4) Apoio das pessoas que trabalham na } \\
\text { empresa/escritório }\end{array}$ & 0,003 & $\mathbf{0 , 9 2 1}$ & 0,047 & $-0,059$ & 0,006 \\
\hline $\begin{array}{l}\text { 13.1) Sua pretensão de adotar internet banda larga } \\
\text { 13.2) Sua expectativa de efetivamente adotar } \\
\text { internet banda larga }\end{array}$ & 0,143 & $-0,132$ & $-0,0051$ & $-0,072$ & $-0,051$ \\
\hline $\begin{array}{l}\text { 13.3) Seus planos para contratar um serviço de } \\
\text { internet banda larga }\end{array}$ & 0,126 & $-0,022$ & 0,161 & 0,221 & $\mathbf{0 , 9 0 8}$ \\
\hline
\end{tabular}

Fonte: Elaborado pelos autores.

Os resultados dos testes foram todos satisfatórios:

- Teste de esfericidade de Bartlett: $\mathrm{H}_{0}$ rejeitada (conforme esperado);

- MSA: das 19 variáveis analisadas, 16 obtiveram índice superior a 0,8 e 3 obtiveram índice superior a 0,7 ;

- KMO: 0,86, superior ao mínimo recomendado de 0,6 (Tabachnick \& Fidell, 2001, p. 589).

Como resultados, foram definidos cinco fatores, sendo a relação entre variáveis e fatores perfeitamente alinhada com a teoria. Ou seja, cada fator gerado estatisticamente coincide exatamente com um dos cinco construtos propostos na UTAUT (Fator 1 - Expectativa de esforço, Fator 2 Influência social, Fator 3 - Expectativa de desempenho, Fator 4 - Condições facilitadoras e Fator 5 Intenção de comportamento).

Nas análises seguintes, foram utilizados os escores fatoriais, criados pelo método de regressão (pelo software SPSS), que gera variáveis padronizadas com média igual a 0 e desvio padrão igual a 1.

Complementando a avaliação da escala adotada, Urdan (1995, p. 298) sugere o uso do coeficiente Alpha de Cronbach para verificação de sua fidedignidade. Segundo Hair et al. (2009, p. 126), esse coeficiente significa: "Medida comumente usada de confiabilidade para um conjunto de dois ou mais indicadores de um construto. Os valores variam entre 0 e 1 , com valores mais altos indicando maior confiabilidade entre indicadores."

Segundo Cooper e Schindler (citado em Sobreira, 2006, p. 116), esse coeficiente “[...] é capaz de revelar o grau em que os itens de um instrumento são homogêneos e refletem o mesmo construto implícito".

Os resultados encontrados (Tabela 2) reforçam a conclusão da análise fatorial de adequação entre as 19 variáveis e os cinco construtos. Em alguns casos, o coeficiente Alpha de Cronbach seria ligeiramente mais elevado com a exclusão de uma variável. Porém, por serem elevações muito 
pequenas, preferiu-se manter todas as variáveis em alinhamento com a teoria original (UTAUT). Na prática, outros autores também optam por manter as variáveis originais do modelo em situações semelhantes (ex.: Sobreira, 2006, p. 120).

Tabela 2 - Avaliação da fidedignidade da escala com o coeficiente Alpha de Cronbach

\begin{tabular}{|l|c|}
\hline \multicolumn{1}{|c|}{ Dimensão } & Coeficiente Alpha da dimensão \\
\hline 1 - Expectativa de esforço & 0,9545 \\
\hline 2 - Influência social & 0,8931 \\
\hline 3 - Expectativa de desempenho & 0,8696 \\
\hline 4 - Condições facilitadoras & 0,9351 \\
\hline 5 - Intenção de comportamento & 0,9698 \\
\hline
\end{tabular}

Fonte: Elaborado pelos autores.

Tabela 2 - Avaliação da fidedignidade da escala com o coeficiente Alpha de Cronbach

\begin{tabular}{|l|l|c|c|c|}
\hline \multicolumn{2}{|l|}{} & $\begin{array}{c}\text { Usuário } \\
\text { banda larga }\end{array}$ & $\begin{array}{c}\text { Não usuário } \\
\text { banda larga }\end{array}$ & Total geral \\
\hline \multicolumn{2}{|l|}{ Dimensão da amostra } & 103 & 104 & 207 \\
\hline Expectativa de esforço & Média & 0,184 & $-0,164$ & 0,009 \\
\hline & Desvio padrão & 0,621 & 1,220 & 0,983 \\
\hline Influência social & Média & $-0,297$ & 0,336 & 0,021 \\
\hline & Desvio padrão & 0,936 & 0,960 & 0,998 \\
\hline Expectativa de desempenho & Média & 0,038 & $-0,061$ & $-0,012$ \\
\hline & Desvio padrão & 0,781 & 1,175 & 0,997 \\
\hline Condições facilitadoras & Média & 0,246 & $-0,210$ & 0,017 \\
\hline & Desvio padrão & 0,776 & 1,066 & 0,958 \\
\hline Intenção de comportamento & Média & 0,584 & $-0,507$ & 0,036 \\
\hline & Desvio padrão & 0,433 & 1,055 & 0,974 \\
\hline
\end{tabular}

Fonte: Elaborado pelos autores.

O levantamento de estatísticas descritivas sobre os cinco fatores (média e desvio padrão) buscou identificar diferenças entre os grupos de usuários e não usuários de banda larga. A simples análise dessas estatísticas descritivas (Tabela 3) já ressalta duas propriedades interessantes:

- Na coluna de total geral observam-se valores de média muito próximos de $\mathbf{0}$ e valores de desvio padrão muito próximos de 1. Isso se deve à própria definição do método de geração dos fatores, que tem como resultados variáveis padronizadas.

- Em quatro dos cinco fatores, a média no grupo de usuários de banda larga é maior que a média de não usuários (a exceção fica para a influência social). Observe-se que o fator Expectativa de esforço é formado de frases que traduzem graus de facilidade com a nova tecnologia. Logo, quanto maior a média, maior o grau de facilidade e menor o esforço esperado. As médias maiores de quatro fatores para o grupo de usuários de banda larga indicam - como já era de se esperar - que as barreiras dos já usuários de banda larga são 
menores do que para os não usuários de banda larga. No entanto, essas diferenças ainda devem ser testadas estatisticamente.

A técnica de análise de variância multivariada (MANOVA) foi aplicada com o objetivo de identificar diferenças significativas de média dos cinco fatores da UTAUT, em conjunto, para os dois grupos focalizados. Como resultado da MANOVA, observou-se que, no conjunto das cinco variáveis, há diferenças significativas entre os dois grupos. Essa conclusão pode ser tomada pelas quatro estatísticas resultantes da técnica: Pillai’s Trace, Wilk's Lambda, Hotelling's Trace e Roy's Largest Root, conforme a Tabela 4.

\section{Tabela 4 - MANOVA (testes multivariados)}

\begin{tabular}{|l|c|c|c|c|}
\hline \multicolumn{1}{|c|}{ Estatística } & Valor & F & P-value & Poder observado \\
\hline Pillai's Trace &, 501 & 40,362 &, 000 & 1,000 \\
\hline Wilks' Lambda &, 499 & 40,362 &, 000 & 1,000 \\
\hline Hotelling's Trace & 1,004 & 40,362 &, 000 & 1,000 \\
\hline Roy's Largest Root & 1,004 & 40,362 &, 000 & 1,000 \\
\hline
\end{tabular}

Fonte: Elaborado pelos autores.

Dado que a MANOVA identificou que, no conjunto dos cinco fatores, há diferença entre os dois grupos pesquisados, testes-t foram realizados para identificar os fatores individuais que se diferenciam entre os dois grupos. Para uma MANOVA de dois grupos recomenda-se o uso da estatística $\mathrm{T}^{2}$ de Hotelling. Esse procedimento garante que a probabilidade de qualquer erro Tipo I ao longo de todos os testes se mantém no nível de significância especificado no cálculo de $\mathrm{T}^{2}$ crit.

A fórmula para cálculo de $\mathrm{T}^{2}$ crit é dada a seguir (Hair et al., 2009, p. 311). Nessa fórmula $\mathrm{p}$ equivale ao número de variáveis dependentes, e Fcrít é definido com p e $(\mathrm{N} 1+\mathrm{N} 2-2-1)$ graus de liberdade. Neste estudo, $\mathrm{p}=5, \mathrm{~N} 1=107, \mathrm{~N} 2=112$, Fcrit $=2,21$, considerando-se nível de significância de 0,05 .

$$
T_{c r i t}^{2}=\frac{p \cdot\left(N_{1}+N_{2}-2\right)}{N_{1}+N_{2}-p-1} F_{c r i t}
$$

A Tabela 5 indica que os fatores intenção de comportamento, influência social e condições facilitadoras se diferenciam entre os dois grupos para o valor 3,36 para Tcrít. 
Tabela 5 - Teste $\mathbf{T}^{2}$ de Hotelling

\begin{tabular}{|l|c|}
\hline \multicolumn{1}{|c|}{ Fator } & $\mathbf{t}_{\text {observ }}$ \\
\hline Expectativa de esforço & $-2,589$ \\
\hline Influência social & 4,806 \\
\hline Expectativa de desempenho & $-0,718$ \\
\hline Condições facilitadoras & $-3,524$ \\
\hline Intenção de comportamento & $-9,743$ \\
\hline
\end{tabular}

Fonte: Elaborado pelos autores.

Por meio do teste de igualdade de médias de cinco fatores ao longo de dois grupos (usuários e não usuários de banda larga) foi possível detectar que os não usuários apresentam:

- menor predisposição de adotar a tecnologia de banda larga em comparação à intenção de continuação de uso declarada pelos adeptos desse recurso;

- maior suscetibilidade à recomendação de terceiros de que deveriam usar a nova tecnologia;

- menor percepção de existência de uma infraestrutura organizacional e técnica para suportar o uso da nova tecnologia.

\section{CONCLUSÕES}

A motivação de desenvolvimento deste trabalho deveu-se à constatação de crescimento acelerado do mercado de banda larga no Brasil e da falta de produtos de banda larga específicos para pequenas empresas. Nesse contexto, este estudo foi desenvolvido com o propósito de:

- testar a dimensionalidade e a fidedignidade de um modelo de aceitação tecnológica no contexto de adoção de banda larga;

- identificar os fatores do modelo que apresentam diferenças significativas entre os usuários e não usuários de banda larga.

Sobre as variáveis da teoria estudada (UTAUT), a avaliação da dimensionalidade da escala (por meio de análise fatorial) indicou total adequação entre as 19 variáveis do questionário e os cinco construtos teóricos. Essa adequação foi verificada também na avaliação da fidedignidade da escala (por meio do coeficiente Alpha de Cronbach).

Em seguida, foram identificadas diferenças nos construtos entre os dois grupos pesquisados.

Foram identificadas diferenças significativas para os fatores intenção de comportamento, influência social e condições facilitadoras. Dentre esses fatores, os que apresentam média negativa 
(condições facilitadoras e intenção de comportamento) para os não usuários de banda larga podem representar barreiras de adoção de internet banda larga por parte do potencial cliente.

Chamou a atenção o fato de a influência social ter apresentado valores mais elevados para os não usuários de banda larga (ao contrário de todos os outros fatores). Em princípio, isso indicaria que a influência social é uma barreira para os já usuários de banda larga (o que não faz muito sentido, dado que já são usuários de banda larga). No entanto, outra interpretação mais coerente é que, entre os usuários de banda larga - justamente por já possuírem banda larga -, a influência social não se manifesta, ocorrendo o contrário entre os não usuários.

A Tabela 1 explicitou as variáveis dentro de cada construto. As variáveis que compõem os fatores com média negativa para os não usuários de banda larga, sobretudo os fatores que distinguiram estatisticamente os dois grupos pesquisados, podem ser utilizadas como referências a serem analisadas com mais profundidade pelas fornecedoras do serviço de internet banda larga para pequenas empresas que pretenderem reduzir as barreiras de adoção desse serviço.

\section{REFERÊNCIAS}

Ajzen, I. (1991). The theory of planned behavior. Organizational Behavior and Human Decision Processes, 50(2), 179-211.

Ajzen, I., \& Fishbein, M. (1980). Understanding attitudes and predicting social behavior. New Jersey: Prentice-Hall.

Bandura, A. (1986). Social foundations of thought and action. New Jersey: Prentice Hall.

Brown, S. A., Dennis, A. R., \& Venkatesh, V. (2010). Predicting collaboration technology use: integrating technology adoption and collaboration research. Journal of Management Information Systems, 27(2), 9-54.

Comitê Gestor da Internet no Brasil. (n.d.). A evolução da Internet no Brasil. Recuperado em 10 de outubro, 2011, de http://www.cgi.br/publicacoes/index.htm.

Compeau, D., \& Higgins, C. (1995) Computer self-efficacy: development of a measure and initial test. MIS Quarterly, 19(2), p. 189-211.

Compeau, D., Higgins, C., \& Huff, S. (1999). Social cognitive theory and individual reactions to computing technology: a longitudinal study. MIS Quarterly, 23(2), p. 145-158.

Davis, F. (1989). Perceived usefulness, perceived ease of use, and user acceptance of information technology. MIS Quarterly, 13(3), 319-340. 
Davis, F., Bagozzi, R., \& Warshaw, P. (1989). User acceptance of computer technology: a comparison of two theoretical models. Management Science, 35(8), 982-1003.

Davis, F., Bagozzi, R., \& Warshaw, P. (1992). Extrinsic and intrinsic motivation to use computers in workplace. Journal of Applied Social Psychology, 22(14), 1111-1132.

Fishbein, M., \& Ajzen, I. (1975) Belief, attitude, intention and behavior: an introduction to theory and research. Massachusetts: Addison-Wesley.

Hair, J. F., Jr., Black, W. C., Babin, B. J., Anderson, R. E., \& Tatham, R. L.(2009). Análise multivariada de dados (A. S. Sant'Anna, Trad.) (6a ed.). São Paulo: Bookman.

Instituto Brasileiro de Geografia e Estatística. (2003). As micro e pequenas empresas comerciais e de serviços no Brasil. Rio de Janeiro: Autor.

Moore, G. C., \& Benbasat, I. (1991). development of an instrument to measure the perceptions of adopting an information technology innovation. Information Systems Research, 2(3), 192-222.

Rogers, E. (1995). Diffusion of innovations ( $4^{\text {th }}$ ed.). New York: Free Press.

Rogers, E., \& Shoemaker, F. (1971). Communications of innovations: a cross-cultural approach. New York: Free Press.

Sharma, S. (1996). Applied multivariate techniques. New York: John Willey \& Sons.

Sobreira, F., Netto. (2006). Medição de desempenho do gerenciamento de processos de negócioBPM no PNAFE: uma proposta de modelo. Tese de Doutorado. Faculdade de Economia, Administração e Contabilidade da Universidade de São Paulo, São Paulo, SP, Brasil.

Tabachnick, B., \& Fidell, L. (2001). Using multivariate statistics ( $4^{\text {th }}$ ed.). Boston: Allyn \& Bacon.

Taylor, S., \& Todd, P.A. (1995). Understanding information technology usage: a test of competing models. Information Systems Research, 6(4), 144-176.

Telebrasil. (n.d.). Situação da banda larga no Brasil: avaliação do diagnóstico realizado pelo IPEA. Recuperado em 10 de outubro, 2011, de http://www.telebrasil.org.br/pnbl_sinditelebrasil_teleco_situacao_banda_larga_no_brasil.pdf.

Teo, T. (2011). Continuing the intention-usage debate in technology acceptance research. International Journal of Instructional Media, 38(4), 327-335.

Thompson, R., Higgins, C., \& Howell, J. (1991). Personal computing: toward a conceptual model of utilization. MIS Quarterly, 15(1), 124-143.

Urdan, A. T. (1995). Avaliação empírica da escala SERVQUAL em serviços hospitalares: uma crítica e réplica ao estudo de Vinícius Sittoni Brasil. Anais do Encontro da Associação Nacional de PósGraduação e Pesquisa em Administração, João Pessoa, PB, 19.

Venkatesh, V., Morris, M., Davis, G., \& Davis, F. (2003). User acceptance of information technology: toward a unified view. MIS Quarterly, 27(3), 425-478. 
Wu, C. S., Cheng, F. F., Yen, D. C., \& Huang, Y. W. (2011). User acceptance of wireless technology in organizations: a comparison of alternative models. Computer Standards \& Interfaces, 33(1), 5058.

\title{
DISCRIMINANT ASPECTS BETWEEN USER AND NON-USERS OF BROADBAND INTERNET
}

\begin{abstract}
The internet has been detached as communication means in many different ways. This development would not be possible if data transmission technology through the internet had not been developed enough to allow adequate speed for these applications. In small business markets, one can still find a significant part of dial-up internet users that have not yet adopted broadband. This study had two objectives: test the dimensionality and the reliability of a technology acceptance model in the context of broadband adoption and identify the factors in the model that have significant differences between users and non-users of this service. Significant differences have been identified for the factors: behavior intention, social influence and facilitating conditions. Among these factors, the ones that present negative means (facilitating conditions and behavior intention) for the non-users of broadband may represent barriers in broadband adoption by the potential client.
\end{abstract}

Key words: Technology acceptance; Broadband Internet; Small businesses.

Data do recebimento do artigo: 03/04/2012

Data do aceite de publicação: 01/08/2012

Revista de Administração e Inovação, São Paulo, v. 9, n.3, p. 195-212, jul/set. 2012. 